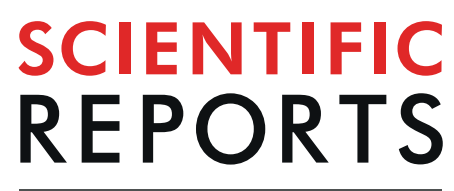

natureresearch

\title{
OPEN Comprehensive analysis of circRNAs from cashmere goat skin by next generation RNA sequencing (RNA-seq)
}

Yuanyuan Zheng ${ }^{1}$, Taiyu $\mathrm{Hui}^{1}$, Chang Yue ${ }^{1}$, Jiaming Sun ${ }^{1}$, Dan Guo ${ }^{2}$, Suling Guo ${ }^{3}$, Suping Guo ${ }^{1}$, Bojiang $\mathrm{Li}^{1}$, Zeying Wang ${ }^{1 *}$ \& Wenlin Bai ${ }^{1 *}$

Circular RNA (circRNA) is endogenous non-coding RNA (ncRNA) with a covalently closed circular structure. It is mainly generated through RNA alternative splicing or back-splicing. CircRNA is known in the majority of eukaryotes and very stable. However, knowledge of the circRNA involved in regulating cashmere fineness is limited. Skin samples were collected from Liaoning cashmere goats (LCG) and Inner Mongolia cashmere goats (MCG) during the anagen period. For differentially expressed circRNAs, RNA sequencing was performed, and the analysis led to an identification of 17 up-regulated circRNAs and 15 down-regulated circRNAs in LCG compared with MCG skin samples. In order to find the differentially expressed circRNAs in LCG, we carried out qPCRs on 10 candidate circRNAs in coarse type skin of LCG (CT-LCG) and fine type skin of LCG (FT-LCG). Four circRNAs: ciRNA128, circRNA6854, circRNA4154 and circRNA3620 were confirmed to be significantly differential expression in LCG. Also, a regulatory network of circRNAs-miRNAs was bioinformatically deduced and may help to understand molecular mechanisms of potential circRNA involvement in regulating cashmere fineness.

The goat (Capra hircus), is economically important livestock, used in the production of cashmere, meat, and milk. The Liaoning cashmere goat breed (LCG) is famous for high fiber production ${ }^{1}$, whereas Inner Mongolia cashmere goats (MCG) produce high-quality cashmere fiber compared with other cashmere goat breeds ${ }^{2}$. In recent years, the characteristics of cashmere fiber have received special attention in that they play an obvious role in cashmere quality. Several studies indicated that coding and noncoding genes were associated with the regulation of cashmere growth ${ }^{3-5}$. In addition, several important pathways have been demonstrated to be related to the formation of cashmere fiber ${ }^{6-8}$, for instance, Wnt, NF- $\kappa B$, Shh, Notch and other signaling pathways ${ }^{9-13}$. However, there are no systematic studies on the molecular regulation of cashmere fineness in the skin.

Circular RNAs (CircRNAs), a new class in the eukaryotic transcriptome, are characterized by the $3^{\prime}$ and $5^{\prime}$ ends of which are covalently linked in a covalently closed loop without free ends $s^{14,15}$. CircRNAs, with the unique circular structure, are more stable and have longer half-lives than mRNAs ${ }^{16,17}$. More recently, many studies have demonstrated that circRNAs contribute to the generation of cancer ${ }^{18,19}$, regulate gene expression in many biological processes, and participate in the occurrence and development of various diseases ${ }^{20}$. In a study by Li et al. identified 6,113 circRNAs from muscles of sheep by RNA-seq ${ }^{21}$, and a total of 10,226 circRNAs were detected from pituitary glands of sheep by RNA-seq ${ }^{22}$. Zheng et al. revealed that circRNAs can act as a microRNA sponge to isolate microRNA by competing with targeted mRNA ${ }^{23}$. Another investigation determined that 151 circRNAs were differently expressed in ORFV-infected goat skin fibroblast cells and uninfected cells ${ }^{24}$. The mechanism of potential involvement of circRNAs in cashmere formation remains unclear.

\footnotetext{
${ }^{1}$ College of Animal Science \&Veterinary Medicine, Shenyang Agricultural University, Shenyang, 110866, China. ${ }^{2}$ Liaoning Modern Agricultural Production Base Construction Engineering Center, Liaoyang, 111000, China. ${ }^{3}$ Prosperous Community, Changshun Town, Huade, 013350, China. *email: wangzeying2012@syau.edu.cn; baiwenlin@syau.edu.cn
} 

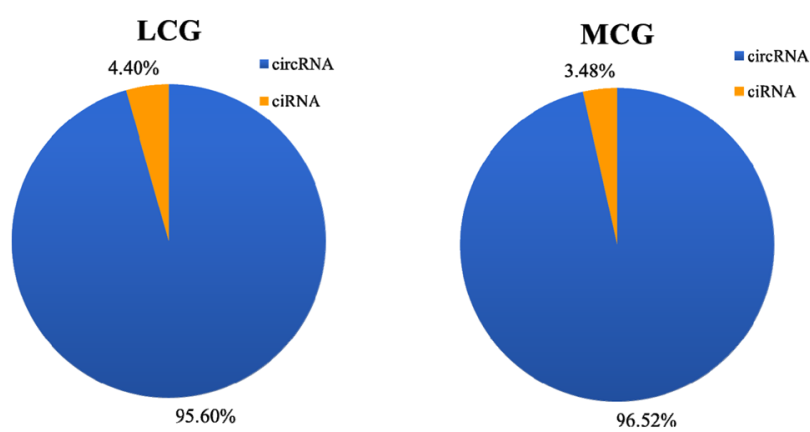

a.
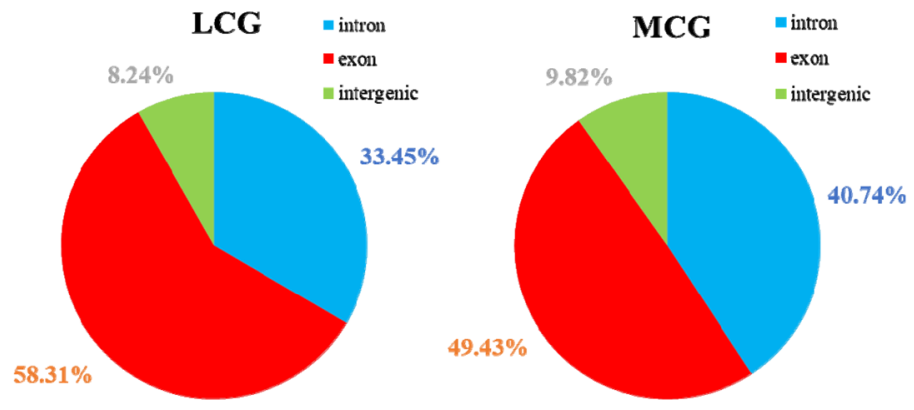

b.

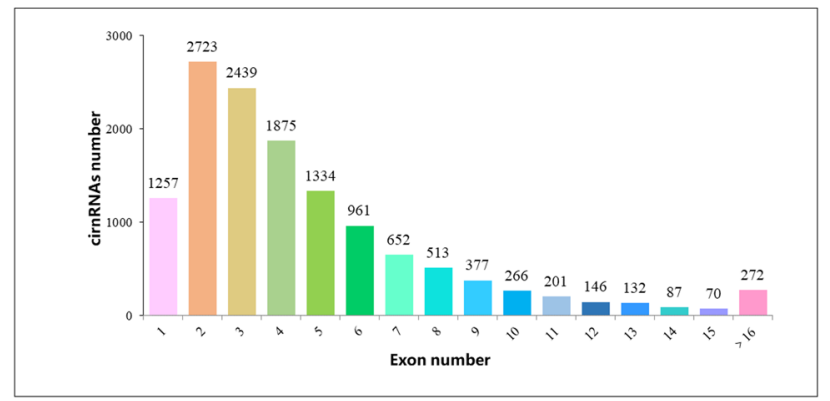

c.

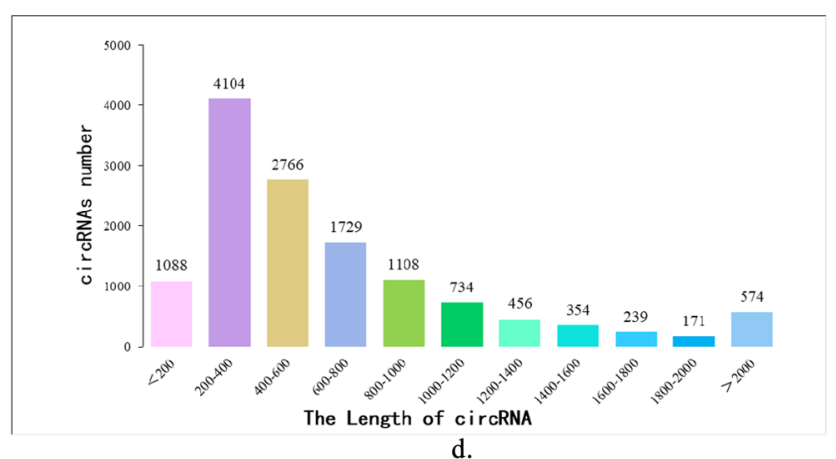

d.

Figure 1. Information on circRNAs from RNA-seq in Liaoning cashmere goats (LCG) and Inner Mongolia cashmere goats (MCG) skin tissue. (a) The types of circRNAs. (b) Distribution of exons, introns, and intergenic circRNAs. (c) The number of exons in circRNAs. (d) The lengths of circRNAs.

In the present study, we aim to find differentially expressed circRNAs in cashmere goat skin. We used RNA-seq to identify the circRNAs in LCG and MCG skin samples, and hundreds of circRNAs were obtained in goat skins. To further explore the relationship between circRNAs with cashmere fineness and its potential role, we also generated a regulatory network that took into account interactions between these circRNAs and miRNAs. Our findings may offer a new insight into cashmere goat circRNAs and their potential involvement in regulation of cashmere fineness. 


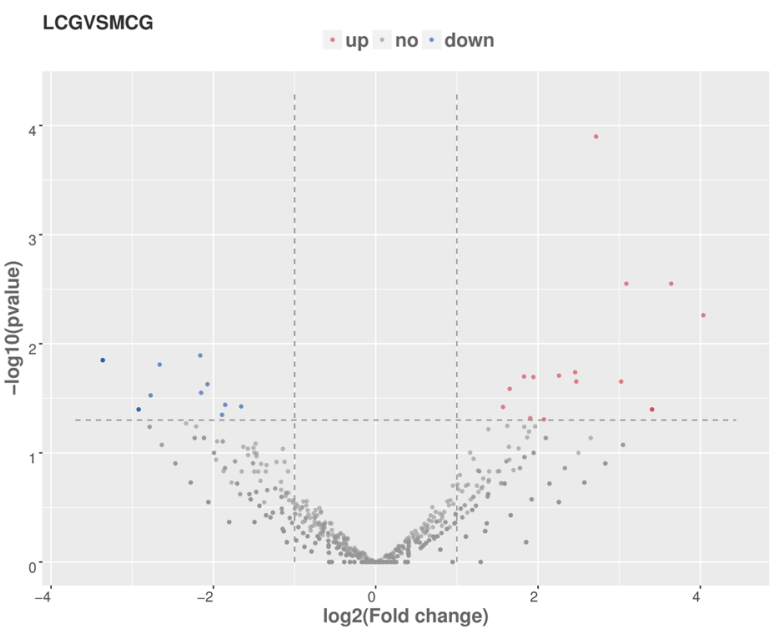

a.

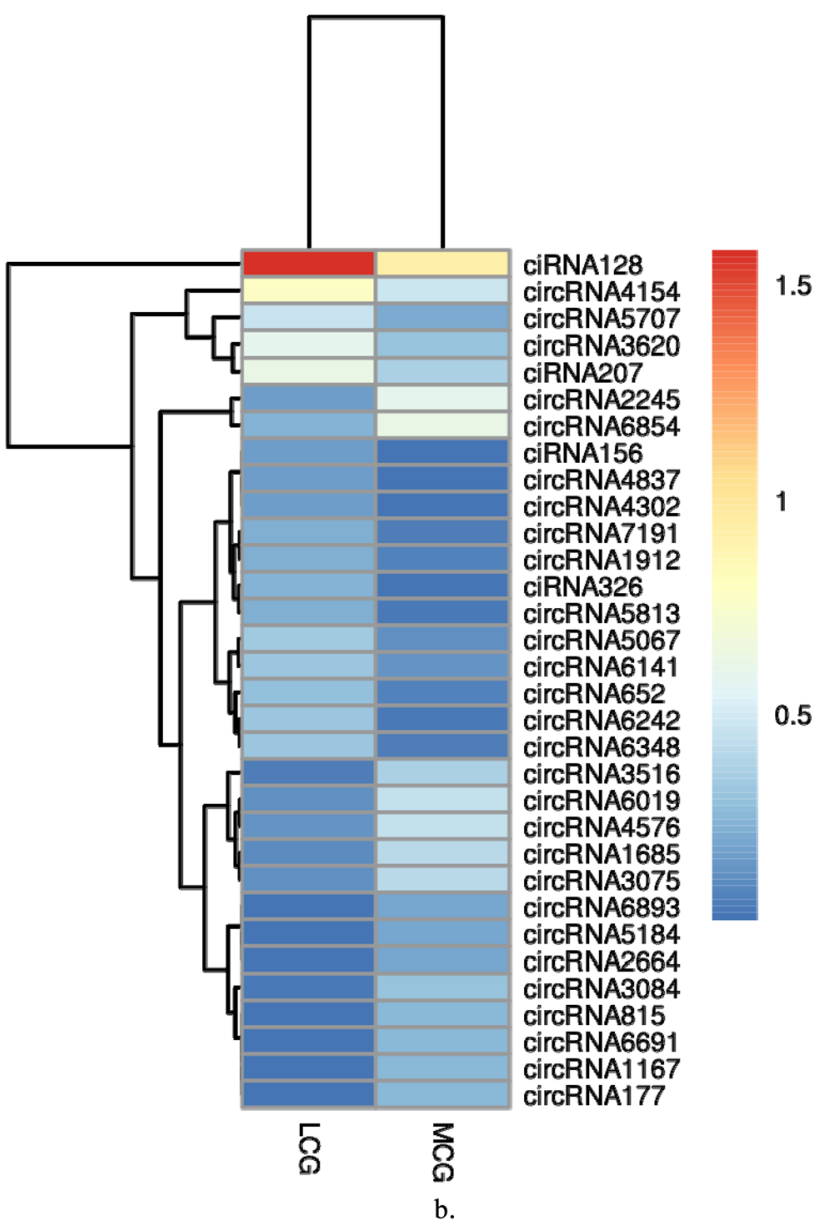

Figure 2. Differentially expressed circRNAs in LCG and MCG. (a) Volcano map of differentially expressed circRNAs. Red dots indicate up-regulation and blue dots indicate down-regulation. (b) Cluster heatmap of differentially expressed circRNAs. The sample is represented by the abscissa and the log value of circRNA expression is regarded by the ordinate, which means that the heatmap is drawn from $\log 10$ of circRNA expression. The highly expressed circRNA is indicated by red, meanwhile, the lowly expressed circRNA is presented by blue.

\section{Results}

Identification of circRNAs in cashmere goat skin. In order to understand the differentially expressed circular RNAs in goat skin, we performed RNA-seq analysis processes in LCG and MCG skin. Total clean reads were obtained after deleting the low-quality raw reads, the mapping ratios of clean reads were $91.27 \%$ and $84.93 \%$ 


\begin{tabular}{|l|l|l|l|l|l|}
\hline circRNA ID & host gene & LCG $($ FPKM $)$ & MCG $($ FPKM $)$ & log2FC & $p$-value \\
\hline ciRNA128 & TCHH & 36.95 & 7.14 & 2.72 & 0.00012611 \\
\hline circRNA4154 & HOMER3 & 5.05 & 2.04 & 1.65 & 0.02588325 \\
\hline ciRNA207 & HCFC1R1 & 3.14 & 1.34 & 1.57 & 0.03802090 \\
\hline circRNA3620 & CAMSAP1 & 2.86 & 1.02 & 1.83 & 0.01997006 \\
\hline circRNA5707 & CREB5 & 1.97 & 0.64 & 1.94 & 0.02020643 \\
\hline circRNA5067 & TMEM62 & 1.24 & 0.32 & 2.26 & 0.01961495 \\
\hline circRNA6242 & SPTBN4 & 1.12 & 0.11 & 3.64 & 0.00281198 \\
\hline circRNA6348 & ARID1A & 1.12 & 0.16 & 3.09 & 0.00281198 \\
\hline circRNA6141 & GSE1 & 1.12 & 0.38 & 1.90 & 0.04803681 \\
\hline circRNA652 & CDC6 & 0.95 & 0.21 & 2.46 & 0.01825840 \\
\hline ciRNA326 & PDLIM2 & 0.79 & 0.05 & 4.04 & 0.00547933 \\
\hline circRNA7191 & PHLPP1 & 0.73 & 0.16 & 2.47 & 0.02220809 \\
\hline circRNA5813 & ISPD & 0.73 & 0.11 & 3.02 & 0.02220809 \\
\hline circRNA1912 & RYK & 0.73 & 0.21 & 2.07 & 0.04933581 \\
\hline circRNA4837 & CASC4 & 0.51 & 0.05 & 3.40 & 0.03997049 \\
\hline circRNA4302 & GFM2 & 0.51 & 0.05 & 3.40 & 0.03997049 \\
\hline ciRNA156 & GRHL1 & 0.51 & 0.05 & 3.40 & 0.03997049 \\
\hline
\end{tabular}

Table 1. Up-regulated circRNAs in LCG and MCG.

\begin{tabular}{|l|l|l|l|l|l|}
\hline circRNA ID & host gene & LCG $($ FPKM $)$ & MCG $($ FPKM $)$ & log2FC & $p$-value \\
\hline circRNA6854 & KCTD9 & 0.79 & 3.17 & -1.66 & 0.03757668 \\
\hline circRNA2245 & HEBP1 & 0.51 & 2.90 & -2.16 & 0.01279541 \\
\hline circRNA4576 & MED17 & 0.39 & 1.82 & -1.85 & 0.03625281 \\
\hline circRNA6019 & AXDND1 & 0.34 & 1.82 & -2.07 & 0.02348295 \\
\hline circRNA3075 & PRPF18 & 0.34 & 1.61 & -1.89 & 0.04479169 \\
\hline circRNA1685 & PHLDB2 & 0.28 & 1.61 & -2.15 & 0.02812314 \\
\hline circRNA3516 & UBXN2A & 0.17 & 1.39 & -2.66 & 0.01551409 \\
\hline circRNA3084 & FAM188A & 0.11 & 1.02 & -2.77 & 0.02972176 \\
\hline circRNA1167 & REV3L & 0.06 & 0.80 & -3.36 & 0.01414588 \\
\hline circRNA177 & BMS1 & 0.06 & 0.80 & -3.36 & 0.01414588 \\
\hline circRNA6691 & PHLDB2 & 0.06 & 0.80 & -3.36 & 0.01414588 \\
\hline circRNA815 & ARL8B & 0.06 & 0.80 & -3.36 & 0.01414588 \\
\hline circRNA5184 & AP3B1 & 0.06 & 0.59 & -2.92 & 0.03997049 \\
\hline circRNA2664 & VPS72 & 0.06 & 0.59 & -2.92 & 0.03997049 \\
\hline circRNA6893 & AGTPBP1 & 0.06 & 0.59 & -2.92 & 0.03997049 \\
\hline
\end{tabular}

Table 2. Down-regulated circRNAs in LCG and MCG.

in LCG and MCG. A total of 13,320 circRNAs were identified from the RNA-seq data, including 610 circular intronic RNAs (ciRNAs) (Fig. 1a). There are 7,531 and 8,943 circRNAs detected in LCG and MCG libraries, respectively. The MCG samples (49.43\%) were compared with the LCG samples (58.31\%), and the percentage of mapped sequence reads that could be aligned with the exon region were significantly lower (Fig. 1b). The lengths of circRNAs ranged from 200 to $400 \mathrm{bp}$ in LCG and MCG (Fig. 1c), and the majority of circRNAs contained 2-7 exons (Fig. 1d). We found that the genomic loci, from which the circRNAs were derived, were over 29 autosomes and $\mathrm{X}$ chromosomes in the two types of samples.

Differentially expressed circRNAs in LCG and MCG. A total of 32 circRNAs were identified as differentially expressed when we compared the data between LCG and MCG skin tissues (Fig. 2a), of which 17 circRNAs were significantly up-regulated and 15 circRNAs were significantly down-regulated in the LCG (Tables 1 and 2). Then, we used a cluster heat-map analysis of differentially expressed circRNAs to better understand their potential relationship (Fig. 2b). To assure the accuracy of RNA-seq strategy, six differentially expressed circRNAs were randomly selected and specific qPCR primers were designed within the circRNAs' junction regions (Fig. 3a). The expression levels of circRNAs determined by qPCR and RNA-seq are highly consistent (Fig. 3b). This meaning the significant reliability of RNA-seq data acquisition and subsequent analysis procedures in this study. 


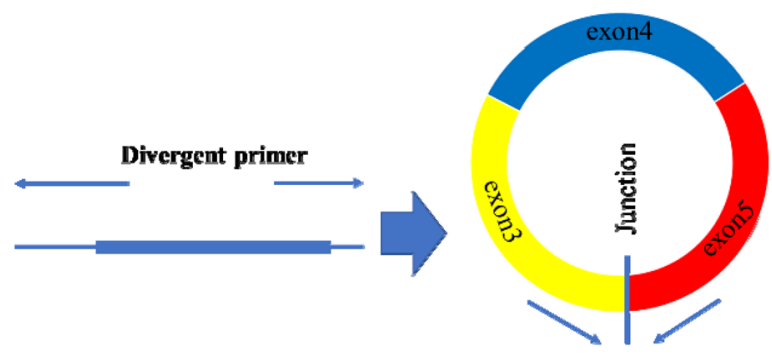

a.

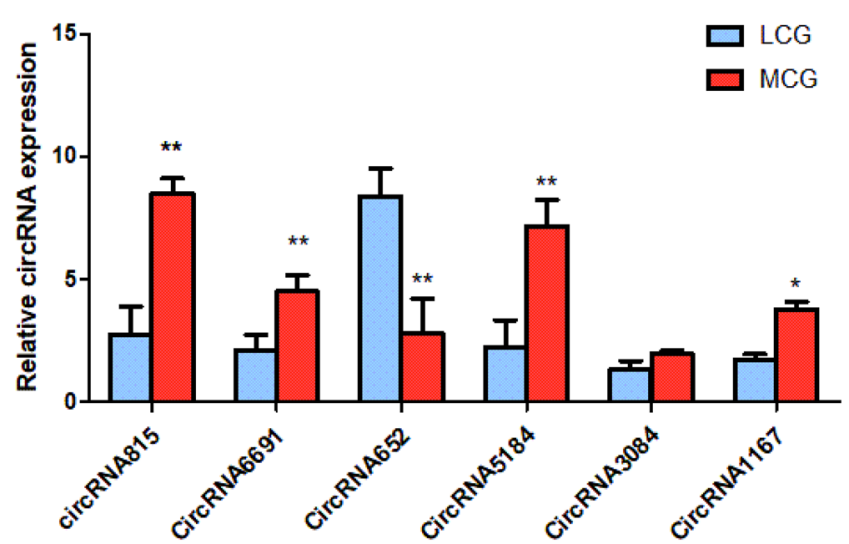

b.

Figure 3. Quantitative real-time PCR (qPCR) result of circRNAs expression. (a) Divergent primers used in the amplification of circular junctions. (b) Validation of putative circRNAs by qPCR. Blue: LCG; Red: MCG. Error bars indicate mean \pm SE for three individuals, “*” $p<0.05$, “**” $p<0.01$.

In our data, total 13,320 circRNAs detected in our study were derived from 4826 host genes. $45 \%$ of these host genes generated only one circRNA, and $20 \%$ of these host genes generated two circRNAs, whereas $8 \%$ of these host genes generated more than six circRNAs (Fig. 4a). We screened 10 candidate circRNAs and found these circRNAs consisted of three or four exons on average (Fig. 4b).

Enrichment analysis of differentially expressed circRNA host genes. We performed GO and KEGG enrichment analysis for the host genes of differentially expressed circRNAs. A total of 22 host genes were enriched in 106 GO terms, and the top 25, top 15 and top 10 in biological processes, cellular components and molecular functions, respectively (Fig. 5a). In Fig. 5b, the top 20 significant GO terms were exhibited, and it can be seen that keratinization and intermediate filament organization were closely related to cashmere fiber growth. A total of 43 pathways were enriched using KEGG analysis, and the top 20 pathways were shown in Fig. 6 . These include the sulfur relay system, sulfur metabolism, and glycosaminoglycan degradation pathways, suggesting that these pathways could also be involved in the regulation of cashmere fineness.

Analysis of interactions between circRNAs and miRNAs. It is generally accepted that circRNA is an adsorbed miRNA sponge and interacts with miRNA. We predicted the potential circRNAs-miRNAs interactions for these differential circRNAs, and the results indicated that the co-expression networks included 32 differentially expressed circRNAs, their host genes and 244 miRNAs (Fig. 7a). The results suggested that circRNA6854 may function as a sponge for these miRNAs, such as chi-miR-106a-5p, chi-miR-106b-5p, chi-miR17-5p, chi-miR-20a-5p, chi-miR-20b, chi-miR-338-3p, chi-miR-378-5p, and chi-miR-93-5p. CiRNA128 has an interaction with chi-miR-331-5p and chi-miR-877-3p. Moreover, the interactions between 10 identified differentially expressed candidate circRNAs and their target miRNAs are presented in Fig. $7 \mathrm{~b}$.

Validation of differentially expressed circRNAs by qPCR. To investigate the expression of circRNAs and determine circRNAs may be vital for regulating cashmere fineness in LCG skin tissue, we used qPCR to confirm the differential expression of certain circRNAs in coarse type and fine type LCG skins. Ten differentially expressed circRNAs were selected and specific qPCR primers were designed within the circRNAs' junction regions. RNA-seq results showed that ciRNA128 had the highest expression level among the up-regulated circRNAs, while circRNA6854 had the highest expression level among the down-regulated circRNAs. The qPCR experiment results of CT-LCG and FT-LCG were shown in Fig. 8. It was proven that these circRNAs really existed and showed similar expression patterns in LCG skin, with the majority exhibiting a higher expression level in FT-LCG. The results of ciRNA128, circRNA6854, circRNA3620 and circRNA4154 are significantly differential expressed in RNA-seq and qPCR, which suggests that they might play a positive role in cashmere goats with different fiber diameters. 


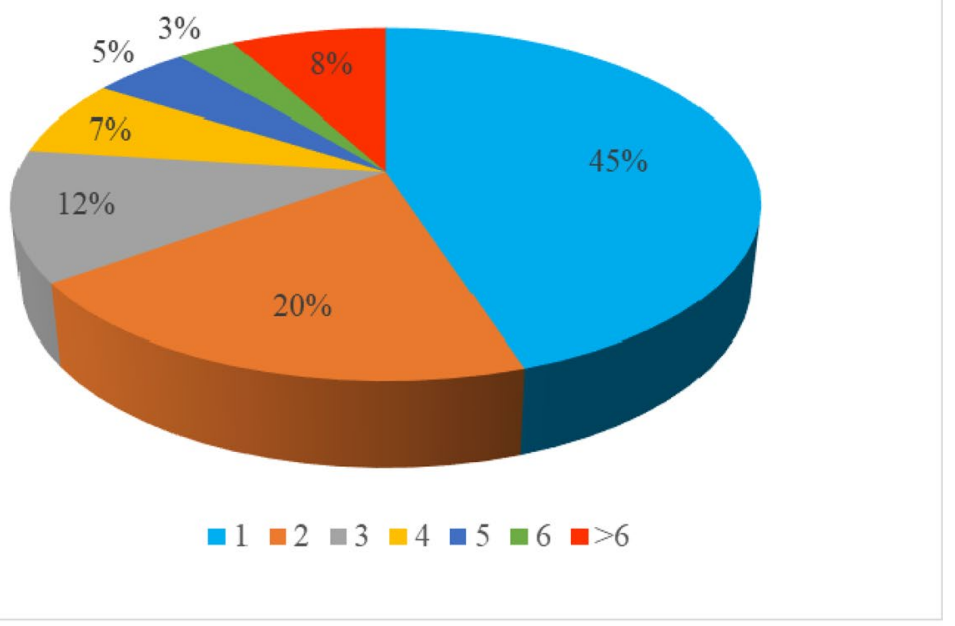

a.

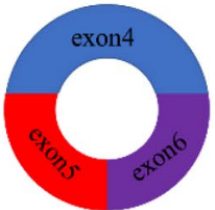

circRNA4154

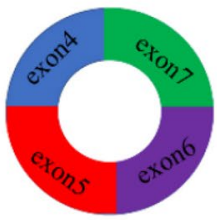

circRNA1685

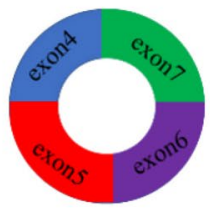

circRNA6854

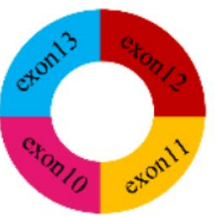

circRNA5067

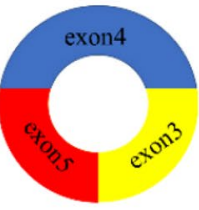

circRNA3620

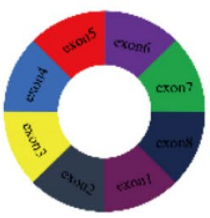

circRNA4576

b.

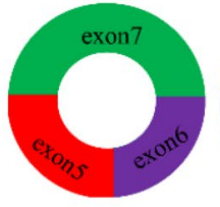

circRNA5707

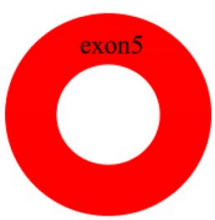

ciRNA128

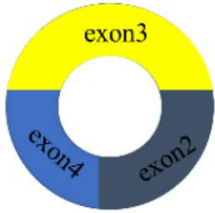

circRNA2245

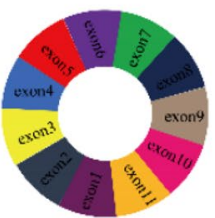

circRNA3075

Figure 4. (a) Numbers of circRNAs produced by the same gene. (b) Exon distribution of candidate circRNAs. Different colors represent different exons.

\section{Discussion}

The cashmere goat is a great breed that produces large amounts of high-grade cashmere fiber. As one of the largest producers of cashmere in the world, China has made tremendous contributions to the world animal fiber industry and plays an indispensable role in global cashmere production ${ }^{25}$. CircRNAs can be classified into four categories: ecRNA, EIciRNA, ciRNA, and tricRNA ${ }^{26}$. CircRNAs located in the nucleus are mainly involved in transcriptional regulation. During the past few years, through high-throughput RNA sequencing and bioinformatics analysis, a great number of circRNAs have been discovered in different species and tissues. For example, 13,950 circRNAs were detected in pre-ovulatory ovarian follicles of goats, and 37 circRNAs were found to be differentially expressed ${ }^{27}$. Empirical Bayes sequencing analysis identified 11 down-regulated and 32 up-regulated circRNAs in embryos with black fur skin and white fur skin of mice, and these circRNAs may play a role in skin pigmentation $^{28}$. The effects of mRNA and lncRNA have been reported on the skin and hair follicles ${ }^{29,30}$, but there are few studies on the effect of circRNAs on the fineness of cashmere and cashmere growth.

In recent years, numerous studies have found that circRNAs located in cytoplasm can compete with mRNAs for target binding sites of miRNAs to regulate the expression of mRNAs. The interaction between circRNA and miRNA has attracted more and more attention. In fact, a number of non-coding RNAs have been identified and reported in cashmere goat $\operatorname{skin}^{31-33}$. In the current study, we identified a total of 13,320 circRNAs in goat skins using RNA-seq analysis. Among these circRNAs, 32 circRNAs were differentially expressed between LCG and MCG skin, and then we randomly selected 6 circRNAs to verify the expression levels by qRT-PCR. The results of RNA-seq and qPCR were almost identical, thereby indicated the reliability of RNA-seq. Additionally we carried out validation on 10 circRNAs in CT- LCG and FT- LCG, interestingly, these circRNAs also had a high expression level in FT-LCG, which may play potential positive role in regulating fiber fineness formation.

We obtained 106 terms from GO enrichment analysis, including 69 biological processes, 17 molecular functions, and 20 cellular components. Keratinization, intermediate filament organization, spindle midzone, Wnt-protein binding, and Wnt-activated receptor activity negative regulation of stress fiber assembly were significant enriched, 


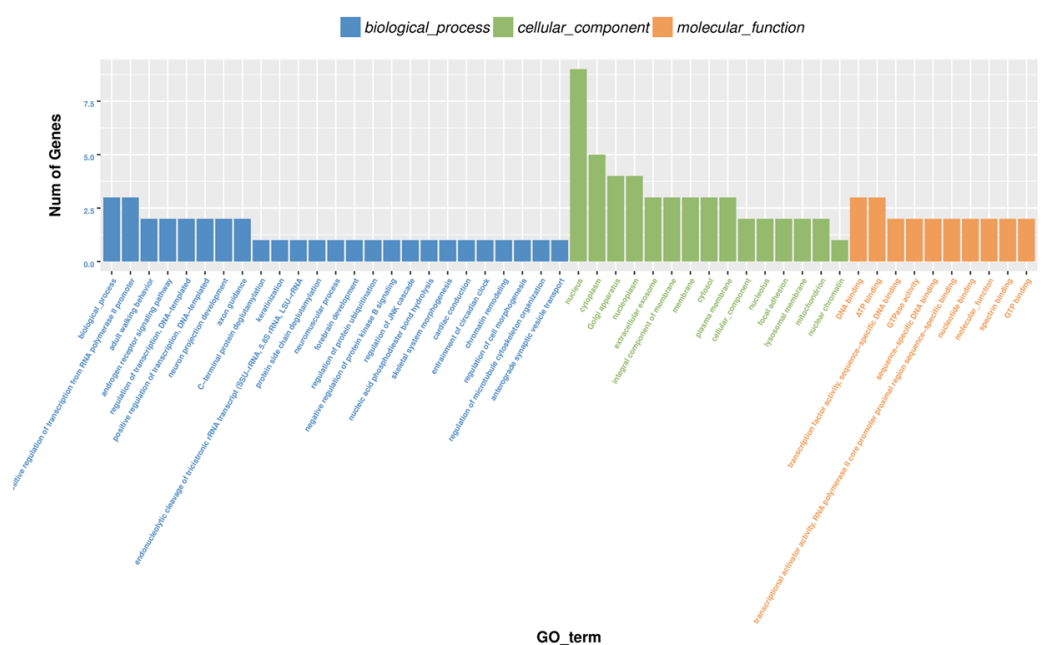

a.

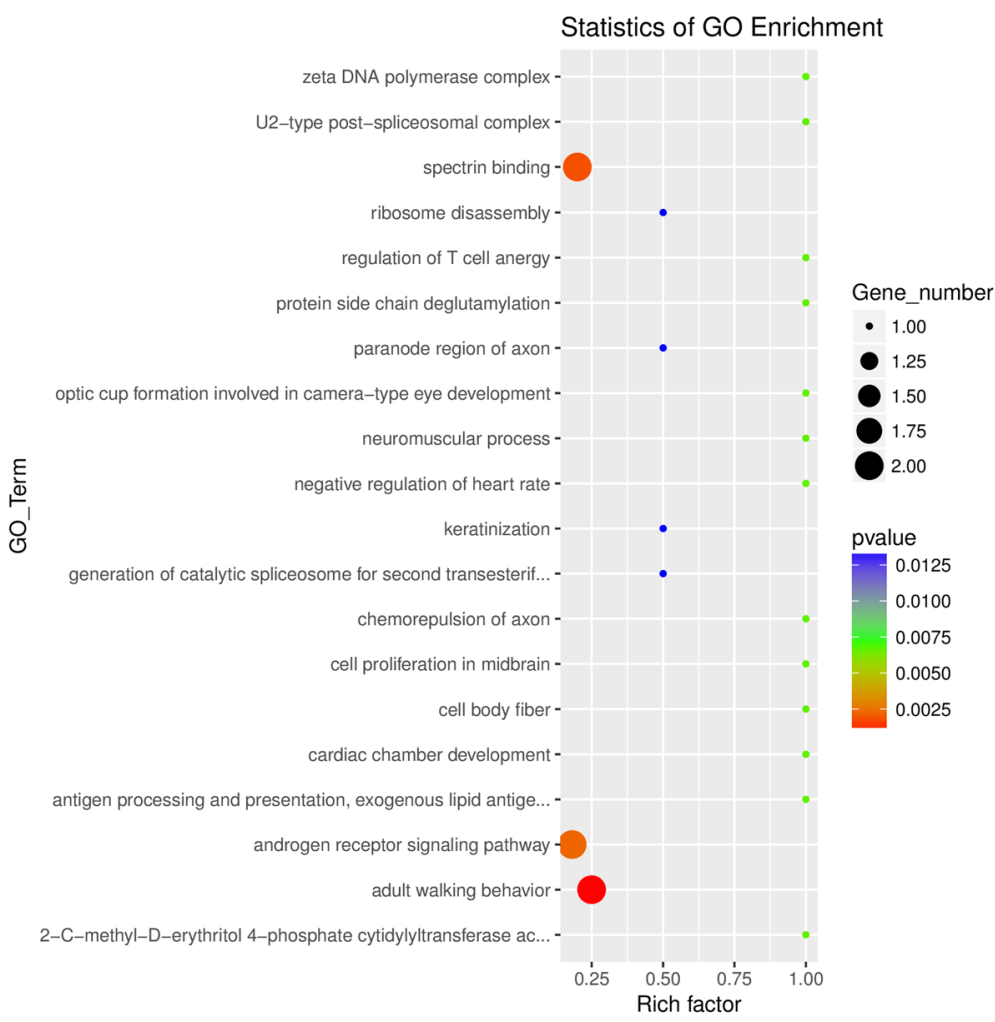

b.

Figure 5. Gene ontology (GO) analysis of differentially expressed circRNAs. (a) Top 25 biological processes, top 15 cell components, and top 10 molecular functions. (b) The top $20 \mathrm{GO}$ terms. The color of the dot corresponds to different $p$-value ranges, and the size of the dot indicates the number of genes in the pathway. Rich factor denotes the number of differentially expressed circRNAs in the GO/ the total number of circRNAs in the GO.

these pathways may participate in the regulation of cashmere fineness formation. Although there were only three pathways in which the sulfur relay system, sulfur metabolism, and glycosaminoglycan degradation were found to be significantly enriched, the host genes of circRNA6854 and circRNA815 were involved in all pathways. Studies have shown that intermediate filaments are probably the key factors involved in cashmere growth ${ }^{34}$. In addition, several important pathways that play a role in dominating hair follicle development were reported, such as the PPAR pathway $^{35}$, Wnt signaling pathway ${ }^{5,36,37}$, MAPK signaling pathway ${ }^{38}$, and NF-kappa B signaling pathway ${ }^{39}$. Our data also enriched these pathways, it further illustrates the importance of these circRNAs in goat skin. 


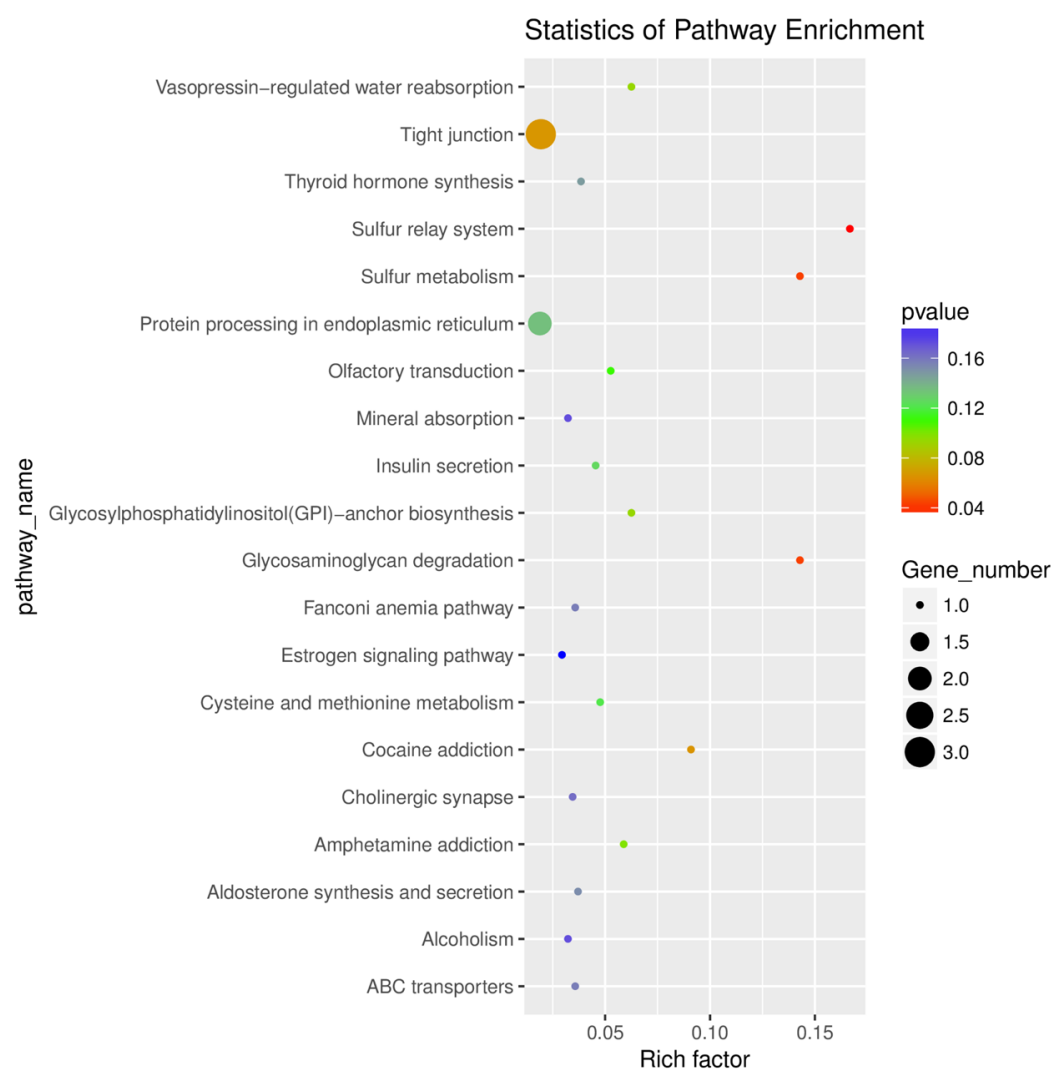

Figure 6. Top 20 Kyoto Encyclopedia of Genes and Genomes (KEGG) pathways of host genes of differentially expressed circRNAs. The color of the dot corresponds to different $p$-value ranges, and the size of the dot indicates the number of genes in the pathway. Rich factor denotes the number of differentially expressed circRNAs in the KEGG/the total number of circRNAs in the KEGG.

Previous studies have shown that circRNA can further influence the expression of target miRNA by acting as a miRNA sponge ${ }^{40-42}$, and the interactions between circRNA and miRNA have been investigated ${ }^{16,43}$. It is noteworthy that some known miRNAs have been reported to be closely related to cashmere growth and development, while some miRNAs may play multiple roles in cashmere goat skin in the growth period. The expressions of mir-103-3p, miR-15b-5p, miR-17-5p, mir-30c-5p, mir-200b, mir-199a-3p, mir-199a-5p, mir-30a-5p, and mir$29 a-3 p$ were significantly different between anagen and telogen skin in Liaoning Cashmere goats ${ }^{44}$, and these were found as the target miRNAs of circRNAs in our data. We hypothesized that a lot of circRNAs interact with many cashmere-related miRNAs (chi-miR-106a-5p, chi-miR-106b-5p, chi-miR-17-5p, let-7b-5p, chi-miR-20b, chi-miR-143). Previous research proved that oar-miR-103-3P, oar-miR-148b-3P, oar-miR-320-3P, oar-miR-31-5P, oar-novel-1-5P, and oar-novel-2-3P may play an important role in follicle growth of Tibetan Sheep ${ }^{45}$. Mir-200b as a target was involved in the regulation of hair follicle development ${ }^{46}$, while miR-1839, miR-374b, and miR-2284n have been reported as showing the highest relative expression levels at the anagen in Inner Mongolia cashmere goat skin tissue ${ }^{33}$. It was reported that let-7b-5p, mir-10a-5p, and mir-21-5p exhibited differences at various hair cycle stages in mouse skin ${ }^{47}$. Research showed that the gene families let-7, mir-17, mir-30, mir-15, and mir- 8 were highly expressed in goat $\operatorname{skin}^{31}$. In Hu sheep lambskin hair follicles, 14 miRNAs including miR-143, miR-10a, and let-7 were screened as important candidate miRNAs ${ }^{48}$. MiR-143, miR-203, and let-7, let-7b, let-7b-5p, let-7f, and let-7c were found to be expressed in Liaoning Cashmere Goats and Fine-Wool Sheep skin ${ }^{49}$, and the let-7 family was reported to be involved in the regulation of cell differentiation ${ }^{50}$. It suggests that let-7b-5p may affect cashmere development as the target of circRNA1167. MiR-378, miR-378e, and miR-378d were only detected in Liaoning Cashmere Goats and promoted angiogenesis ${ }^{50,51}$. Five novel miRNAs (chi-miR-2284n, chi-miR-421*, chi-miR-421, chi-miR-1839, and chi-miR-374) play roles in the production of cashmere in Inner Mongolia cashmere goat skin $^{33}$. Taken together, it can be inferred that ciRNA128-chi-miR-331-5p and circRNA6854-chi-miR17-5p may have certain roles in cashmere fineness and cashmere fiber morphogenesis.

The expression of circRNAs has been appropriately correlated with an abundance of host genes in different animal tissues ${ }^{52-55}$, such as oar_circ_0003451 and TTN, and oar_circ_0005250 and MYH7 may play important roles in muscle development and growth ${ }^{22}$; circRNA8077 and CRIM1, as well as circRNA3314 and TMEM159 play vital roles in the development of the receptive endometrium ${ }^{56}$. The host genes of circRNAs are involved in regulating hair traits, and these circRNAs may be considered as a possible factor regulating cashmere fineness. The host gene TCHH of ciRNA128 has been confirmed to be involved in hair formation ${ }^{57}$. Studies based on GWAs found TCHH in Latin Americans of mixed European and Native American origin ${ }^{58}$. Among Europeans, the strongest link between straight hair and TCHH was found ${ }^{59}$, and DSC2, DSG3, CALML5, TCHH are related 


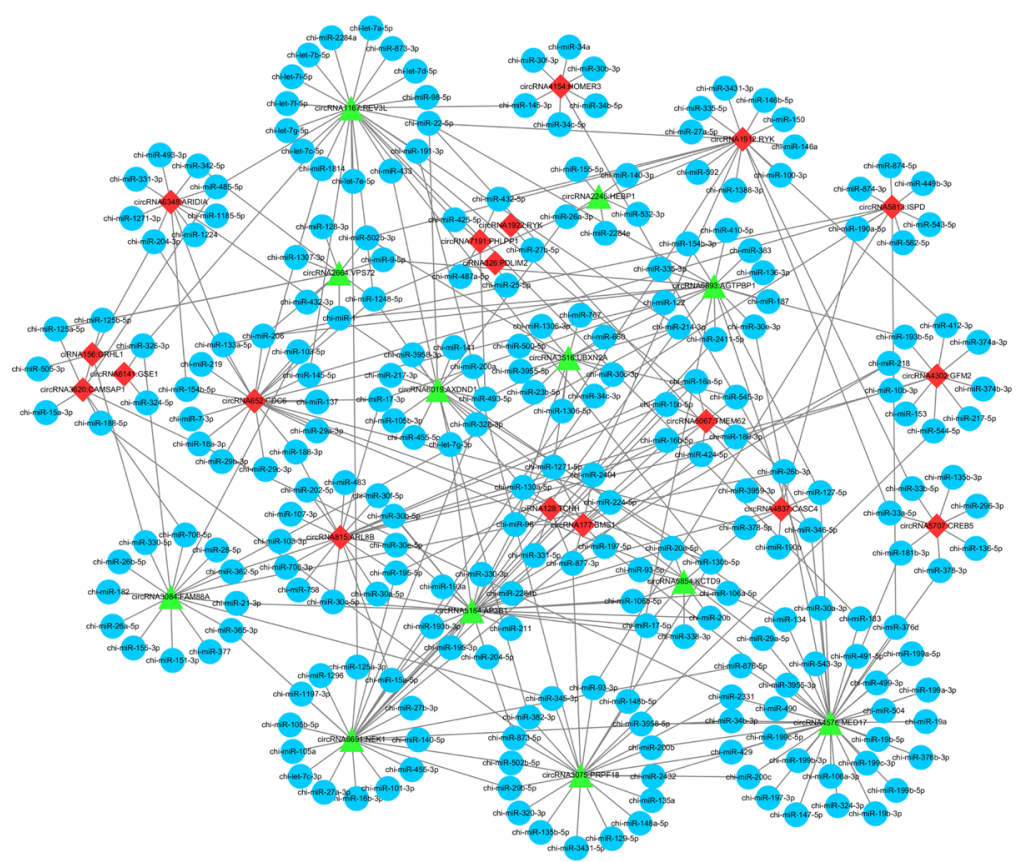

a.

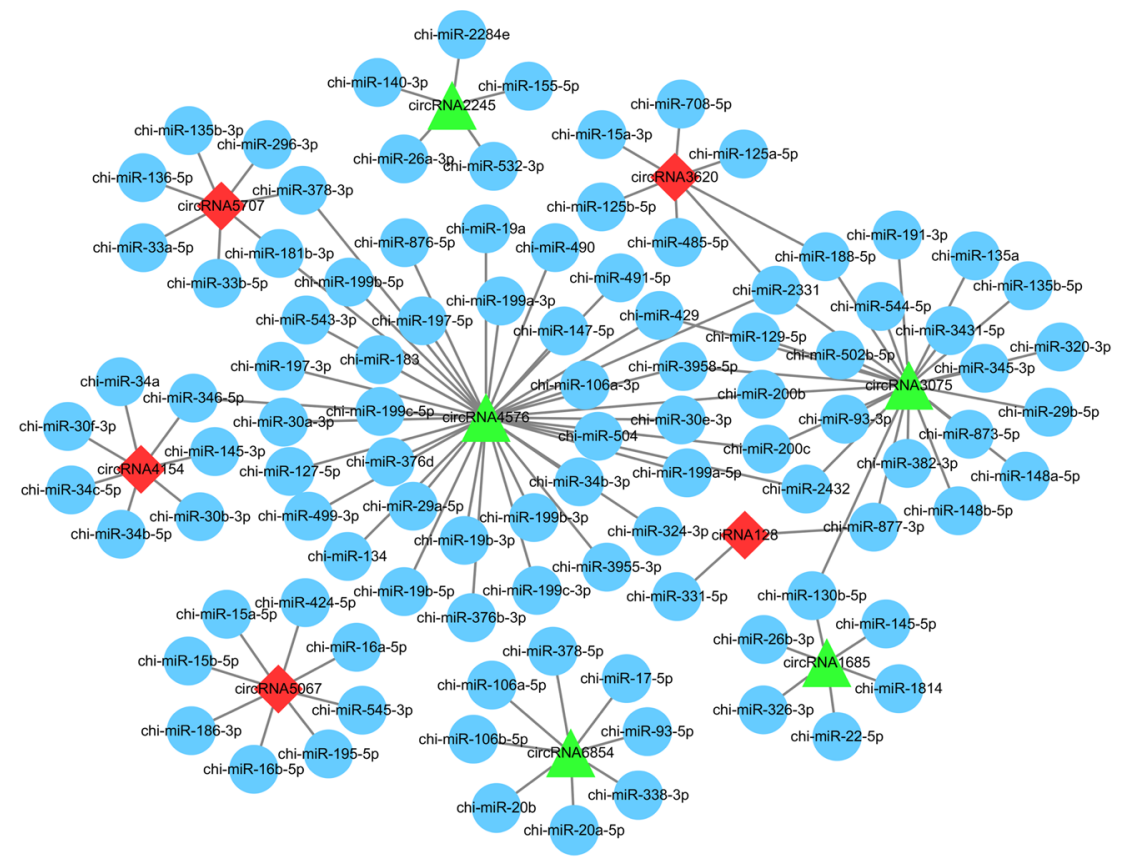

b.

Figure 7. The circRNAs-miRNAs network. (a) Network of 32 differentially expressed circRNAs. Red and green represent up- and down-regulation, and blue represents target-miRNA. (b) The circRNAs-miRNAs network of 10 candidate circRNAs.

to hair growth using iTRAQ-labeling in sheep and goats ${ }^{60}$. KCTD9 has been reported to be associated with can$\operatorname{cer}^{61}$, promoting cell growth and inhibiting cell activation ${ }^{62,63}$. Thus, it proved potential reference value for cashmere fiber fineness and the expression analysis of circRNAs in LCG.

In conclusion, we performed RNA-seq analysis that identified 13,320 circRNAs in cashmere goat skins, of which 32 circRNAs were found to be differential expression. The result of qRT-PCR confirmed that four circRNAs (ciRNA128, circRNA6854, circRNA4154 and circRNA3620) were differentially expressed in CT-LCG and FT-LCG. Host genes of differentially expressed circRNAs were mainly enriched in keratinization and 


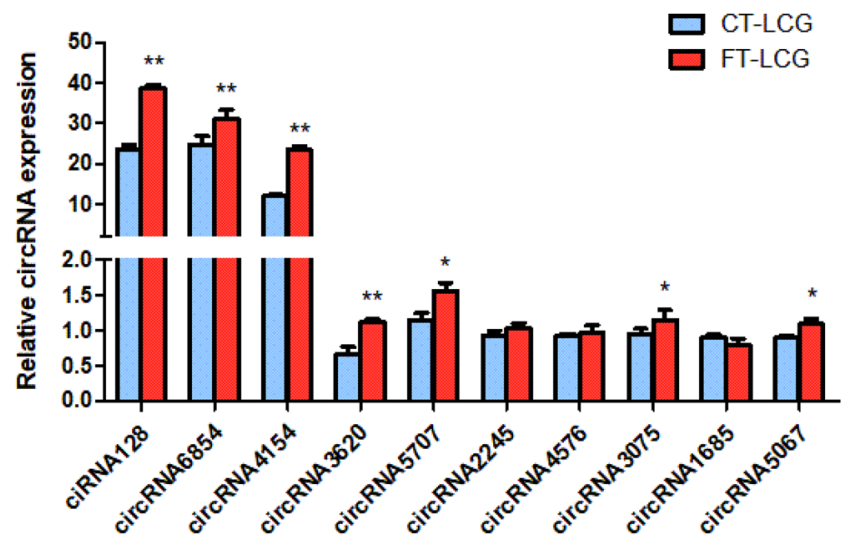

Figure 8. Quantitative real-time PCR results of circRNAs expression. Blue: CT-LCG; Red: FT-LCG. Error bars represents standard deviations within the group, the “*”indicates the significant difference $p<0.05$, “**”indicates $p<0.01$.

intermediate filament organization. An integrated regulatory network of circRNAs and miRNAs was executed in anagen cashmere goat skin. This study may contribute to better understanding of circRNAs in goat skin.

\section{Materials and Methods}

Ethics statement. All experiments in this study were approved and conducted according to the Animal Experimental Committee of Shenyang Agricultural University, Shenyang, China (201606005).

Sample preparation. Skin samples from three adult female Liaoning cashmere goats $(d=19.4 \mu \mathrm{m}, 19.5 \mu \mathrm{m}$ and $19.8 \mu \mathrm{m})$ and three adult female Inner Mongolia cashmere goats $(d=13.8 \mu \mathrm{m}, 14.0 \mu \mathrm{m}$ and $14.1 \mu \mathrm{m})$ were carefully collected. The animals we collected were based on all the same conditions, including sex, age, feeding and physiological status and other factors. To reduce pain to experimental animals, we used local anesthesia with procaine. In the upper one-third of the right scapula along the mid-dorsal and mid-abdominal lines, about $1 \mathrm{~cm}^{2}$ lateral skin from the six cashmere goats were taken and disinfected with $75 \%$ ethanol. And then, the skin samples were washed three times with PBS and immediately stored in liquid nitrogen until RNA isolation. In addition, three coarse type (CT) $\operatorname{skin}(d=19.5 \mu \mathrm{m}, 19.7 \mu \mathrm{m}$ and $20.2 \mu \mathrm{m})$ and three fine type (FT) skin $(d=15.3 \mu \mathrm{m}, 15.4 \mu \mathrm{m}$ and $15.6 \mu \mathrm{m})$ samples from Liaoning cashmere goats were obtained with the same method for qRT-PCR analysis.

Total RNA isolation, library construction and sequencing. The total RNA amount and purity of each sample was quantified by Nano Drop ND-1000 (Nano Drop, Wilmington, DE, USA). Approximately 5 ug of total RNA was used to deplete ribosomal RNA according to the manufacturer's instructions for the Ribo-Zero rRNA Removal Kit (Illumina, San Diego, USA). In order to construct the cDNA library of circRNAs, we used Rnase $\mathrm{R}$ to remove linear RNA. The average insert size for the final cDNA library was $300 \mathrm{bp}( \pm 50 \mathrm{bp})$, the library was purified and qualified by Agilent Bioanalyzer 2100 system.

Identification of circRNAs and analysis of differentially expressed circRNAs. The cDNA libraries were performed the paired-end sequencing on an Illumina Hiseq. 4000 (LC Bio, China) following the vendor's recommended protocol. Firstly, low-quality reads and adapters were removed by Cutadapt v1.10, quality controlled by FastQC v0.10.1, and then obtained the high-quality clean reads. TopHat v2.0.4 was utilized to map the clean reads to the reference genome from National Center for Biotechnology Information (NCBI) (https://www. ncbi.nlm.nih.gov/genome/?term $=$ Capra + hircus $)^{64,65}$. Also, StringTie v1.3.0 was used to assemble and quantify expressed genes and transcripts (https://ccb.jhu.edu/software/stringtie/index.shtml ${ }^{66}$. CIRCExplorer2 v2.2.6 software and the following criteria were used to identify candidate circRNAs: mismatch $\leq 2$, back-spliced junction reads $\geq 1$, and distances of two splice sites of less than $100 \mathrm{~kb}$ in the genome ${ }^{67}$. Then, the back-spliced reads with at least two supporting reads were annotated as circRNAs. The differential expression of circRNAs between the two groups was assessed using the Ballgown package. A $p$-value $<0.05$ and $\mid \log 2$ (fc) $\mid>1$ were set as the threshold for differential expression ${ }^{68,69}$.

Gene ontology (GO) analysis and KEGG analysis of host genes. GO analysis (http://www.geneontology.org) was applied to differentially expressed circRNA-hosting genes. Similarly, pathway analysis uncovered the significant pathways related to differentially expressed circRNAs according to the annotation of the Kyoto Encyclopedia of Genes and Genomes (KEGG) (http://www.kegg.jp/kegg) ${ }^{70}$. A threshold of $p<0.05$ was used as a criterion for the determination of whether the enrichment analysis was significant ${ }^{71}$.

Network construction of the circRNAs-miRNAs interaction. The interaction of circRNAs-miRNAs was predicted with miRNA target prediction software miRanda (http://www.microrna.org/microrna/home.do) and TargetScan (http://www.targetscan.org//72, where the max free energy values of miRanda is $<-10$ and the 
score percentiles of TargetScan is $\geq 50$. The differential expression circRNAs-miRNAs interaction and the network of circRNAs along with their target miRNAs were performed using cytoscape v3.5.1 software (https://cytoscape.org/, USA $)^{73}$.

Quantitative real-time PCR validation. We randomly detected 6 differentially expressed circRNAs for qRT-PCR. To prove the resistance of circRNAs to RNase R digestion, we treated total RNAs with RNase R before cDNA synthesis. In order to validate the differentially expressed circRNAs, total RNAs were synthesized directly to cDNA synthesis by an RT-PCR kit. According to the manufacturer's instructions, Real-time PCR was performed using SYBR Green (TaKaRa Biotech, Dalian). The glyceraldehyde-3-phosphate dehydrogenase (GAPDH) gene was used as an internal control to normalize the expression level of circRNAs ${ }^{74}$. Three independent experiments were carried out on LCG and MCG skin samples. Six pair primers were designed by primer 5 software (www.premierbiosoft.com) and listed in Supplemental Table S1, and all primers were spanning the distal ends of circRNAs. The relative expression levels of different circRNAs were analyzed by the $2^{-\Delta \Delta \mathrm{Ct}}$ method in $\mathrm{qPCR}$ data $^{75}$. The data were indicated as the means $\pm S E(n=3)$. All statistical analyses in the two groups were calculated using a t-test in SPSS statistical software (Version 22.0, Chicago, IL, USA), the difference was significant at $p<0.05$. In addition, three CT-LCG and FT-LCG skin samples were verified by qPCR under the same experimental conditions to find the differential circRNAs in LCG.

Received: 10 October 2019; Accepted: 23 December 2019;

Published online: 16 January 2020

\section{References}

1. Hu, P. F. et al. Study on characteristics of in vitro culture and intracellular transduction of exogenous proteins in fibroblast cell line of Liaoning cashmere goat. Molecular Biology Reports. 40, 327-336 (2013).

2. Zhang, W. G., Wu, J. H., Li, J. Q. \& Midori, Y. A Subset of Skin-Expressed microRNAs with Possible Roles in Goat and Sheep Hair Growth Based on Expression Profiling of Mammalian microRNAs. OMICS: A. Journal of Integrative Biology. 11, 385-396 (2007).

3. Jin, M. et al. Construction of a cDNA library and identification of genes from Liaoning cashmere goat. Livest Sci. 164, 26-34 (2014).

4. Liu, H. et al. Characterization of Liaoning cashmere goat transcriptome: sequencing, de novo assembly, functional annotation and comparative analysis. PLoS One. 8, e77062 (2013).

5. Stenn, K. S. \& Paus, R. Controls of hair follicle cycling. Physiol Rev. 81, 449-494 (2001).

6. Leirós, G. J., Attorresi, A. I. \& Balañá, M. E. Hair follicle stem cell differentiation is inhibited through cross-talk between Wnt/ $\beta$ catenin and androgen signalling in dermal papilla cells from patients with androgenetic alopecia. Br J Dermatol. 166, 1035-42 (2012).

7. Samuelov, L. et al. P-cadherin regulates human hair growth and cycling via canonical Wnt signaling and transforming growth factor-32. J Invest Dermatol. 132, 2332-1241 (2012).

8. Jahoda, C. A. \& Christiano, A. M. Niche crosstalk: intercellular signals at the hair follicle. Cell. 146, 678-681 (2011).

9. Lee, J. \& Tumbar, T. Hairy tale of signaling in hair follicle development and cycling. Semin Cell Dev Biol. 23, 906-916 (2012).

10. Tsai, S. Y. et al. Wnt/beta-catenin signaling in dermal condensates is required for hair follicle formation. Dev Biol. 385, 179-188 (2014).

11. Tripurani, S. K. et al. Suppression of Wnt/ $\beta$-catenin signaling by EGF receptor is required for hair follicle development. Molecular Biology of the Cell. 29, 2784-2799 (2018).

12. Mukhopadhyay, A. et al. Negative regulation of Shh levels by Kras and Fgfr2 during hair follicle development. Dev Biol. 373, 373-382 (2013).

13. Rishikaysh, P. et al. Signaling involved in hair follicle morphogenesis and development. Int J Mol Sci 15, 1647-1670 (2014).

14. Liang, D. \& Wilusz, J. E. Short intronic repeat sequences facilitate circular RNA production. Genes Dev 28, 2233-2247 (2014).

15. Salzman, J., Gawad, C., Wang, P. L., Lacayo, N. \& Brown, P. O. Circular RNAs are the predominant transcript isoform from hundreds of human genes in diverse cell types. Plos One. 7, e30733 (2012).

16. Jeck, W. R. \& Sharpless, N. E. Detecting and characterizing circular RNAs. Nature Biotechnology. 32, 453-461 (2014).

17. Jeck, W. R. et al. Circular RNAs are abundant, conserved, and associated with ALU repeats. Rna-a Publication of the Rna Society. 19, 141-157 (2013).

18. Zhang, H. D., Jiang, L. H., Sun, D. W., Hou, J. C. \& Ji, Z. L. CircRNA: a novel type of biomarker for cancer. Breast Cancer. 25, 1-7 (2018).

19. Yang, R. et al. The circRNA circAGFG1 acts as a sponge of miR-195-5p to promote triple-negative breast cancer progression through regulating CCNE1 expression. Molecular Cancer. 18, 4 (2019).

20. Li, P. et al. Using circular RNA as a novel type of biomarker in the screening of gastric cancer. Clin Chim Acta. 444, 132-136 (2015).

21. Li, G. Y. et al. Genome-wide analysis of circular RNAs in prenatal and postnatal muscle of sheep. Sci Rep. 8, 97165-97177 (2017).

22. Li, G. Y. et al. Genome-wide analysis of circular RNAs in prenatal and postnatal pituitary glands of sheep. Scientific Reports. 7, 16143 (2017).

23. Zheng, Q. P. et al. Circular RNA profiling reveals an abundant circHIPK3 that regulates cell growth by sponging multiple miRNAs. Nature communications. 7, 11215 (2016).

24. Pang, F. et al. Genome-wide analysis of circular RNAs in goat skin fibroblast cells in response to Orf virus infection. PeerJ. 7, e6267 (2019).

25. Su, R. et al. Comparative genomic approach reveals novel conserved microRNAs in Inner Mongolia cashmere goat skin and longissimus dorsi. Mol Biol Rep. 42, 989-995 (2015).

26. Zhang, X. O. et al. Complementary Sequence-Mediated Exon Circularization. Cell. 159, 134-147 (2014).

27. Tao, H. et al. Circular RNA profiling reveals chi_circ_0008219 function as microRNA sponges in pre-ovulatory ovarian follicles of goats (Capra hircus). Genomics. 110, 257-266 (2017)

28. Zhu, Z. et al. Comprehensive circRNA expression profile and construction of circRNA-associated ceRNA network in fur skin. Experimental Dermatology. 27, 3 (2018).

29. Zheng, Y. Y. et al. An Integrated Analysis of Cashmere Fineness IncRNAs in Cashmere Goats. Genes. 10, 4 (2019).

30. Sen, M. et al. Synchronous profiling and analysis of mRNAs and ncRNAs in the dermal papilla cells from cashmere goats. BMC Genomics. 20, 512 (2019).

31. Schmitt, M. J. et al. Interferon-g-induced activation of signal transducer and activator of transcription 1 (STAT1) up-regulates the tumor suppressing microRNA-29 family in melanoma cells. Cell Commun Signal. 10, 41 (2012).

32. Liu, Z. et al. Identification of conserved and novel microRNAs in cashmere goat skin by deep sequencing. Plos One. 7, e50001 (2012).

33. Yuan, C. et al. Discovery of cashmere goat (Capra hircus) microRNAs in skin and hair follicles by Solexa sequencing. BMC Genomics. 14, $511(2013)$. 
34. Zhou, G. X. et al. Integrative analysis reveals ncRNA-mediated molecular regulatory network driving secondary hair follicle regression in cashmere goats. BMC Genomics. 19, 222 (2018).

35. Yue, Y. J. et al. Integrated Analysis of the Roles of Long Noncoding RNA and Coding RNA Expression in Sheep (Ovis aries) Skin during Initiation of Secondary Hair Follicle. Plos One. 11, e0156890 (2016).

36. Bai, W. L. et al. LncRNAs in Secondary Hair Follicle of Cashmere Goat: Identification, Expression, and Their Regulatory Network in Wnt Signaling Pathway. Animal Biotechnology. 29, 1 (2016).

37. Andl, T., Reddy, S. T., Gaddapara, T. \& Millar, S. E. WNT signals are required for the initiation of hair follicle development. Dev Cell. 2, 643-653 (2002)

38. Dickinson, S. E. et al. p38 MAP kinase plays a functional role in UVB-induced mouse skin carcinogenesis. Mol Carcinog. 50, 469-478 (2011).

39. Jin, M. et al. Long noncoding RNA and gene expression analysis of melatonin-exposed Liaoning cashmere goat fibroblasts indicating cashmere growth. Naturwissenschaften (The Science of Nature). 105, 9-10 (2018).

40. Wang, K. et al. A circular RNA protects the heart from pathological hypertrophy and heart failure by targeting miR-223. European Heart Journal. 37, 2602-2611 (2016).

41. Hansen, T. B. et al. Natural RNA circles function as efficient microRNA sponges. Nature. 495, 384-388 (2013).

42. Li, F. et al. Circular RNA ITCH has inhibitory effect on ESCC by suppressing the Wnt/ $\beta$-catenin pathway. Oncotarget. 6, 8 (2015).

43. Yang, W. et al. Foxo3 activity promoted by non-coding effects of circular RNA and Foxo3 pseudogene in the inhibition of tumor growth and angiogenesis. Oncogene. 35, 3919 (2015).

44. Bai, W. L. et al. Differential Expression of microRNAs and their Regulatory Networks in Skin Tissue of Liaoning Cashmere Goat during Hair Follicle Cycles. Animal Biotechnology. 27, 104-112 (2016).

45. Liu, G. B. et al. Identification of microRNAs in Wool Follicles during Anagen, Catagen, and Telogen Phases in Tibetan Sheep. Plos One. 8, e77801 (2013).

46. Andl, T. et al. The miRNA-processing enzyme dicer is essential for the morphogenesis and maintenance of hair follicles. Curr Biol. 16, 1041-1049 (2006).

47. Mardaryev, A. N. et al. Micro-RNA-31 controls hair cycle-associated changes in gene expression programs of the skin and hair follicle. FASEB J. 24, 3869-3881 (2010).

48. Gao, W. et al. Screening candidate microRNAs (miRNAs) in different lambskin hair follicles in Hu sheep. Plos One. 12, e0176532 (2017).

49. Li, J. P. et al. Transcriptome-Wide Comparative Analysis of microRNA Profiles in the Telogen Skins of Liaoning Cashmere Goats (Capra hircus) and Fine-Wool Sheep (Ovis aries) by Solexa Deep Sequencing. DNA and Cell Biology. 35, 696-705 (2016).

50. Deppe, J. et al. Upregulation of miR-203 and miR-210 affect growth and differentiation of keratinocytes after exposure to sulfur mustard in normoxia and hypoxia. Toxicol Lett. 244, 81-87 (2016).

51. Joyce, C. E. et al. Deep sequencing of small RNAs from human skin reveals major alterations in the psoriasis miRNAome. Hum Mol Genet. 20, 4025-4040 (2011).

52. Venø, M. T. et al. Spatio-temporal regulation of circular RNA expression during porcine embryonic brain development. Genome Biol. 16, 245 (2015).

53. Salzman, J., Chen, R. E., Olsen, M. N., Wang, P. L. \& Brown, P. O. Cell-type specific features of circular RNA expression. Plos Genet. 9, e1003777 (2013).

54. Siede, D. et al. Identification of circular RNAs with host gene-independent expression in human model systems for cardiac differentiation and disease. Journal of Molecular and Cellular Cardiology. 109, 48-56 (2017).

55. Rybak-Wolf, A. et al. Circular RNAs in the mammalian brain are highly abundant, conserved, and dynamically expressed. Mol Cell. 58, 870-885 (2015).

56. Song, Y. X. et al. Analyses of circRNA profiling during the development from pre-receptive to receptive phases in the goat endometrium. J Anim Sci Biotechnol. 10, 34 (2019).

57. Liu, F. et al. Meta-analysis of genome-wide association studies identifies 8 novel loci involved in shape variation of human head hair. Human Molecular Genetics. 27, 559-575 (2017).

58. Adhikari, K. et al. A genome-wide association scan in admixed Latin Americans identifies loci influencing facial and scalp hair features. Nat Commun. 7, 10815 (2016).

59. Medland, S. E. et al. Common variants in the trichohyalin gene are associated with straight hair in Europeans. Am J Hum Genet. 85, 750-755 (2009)

60. Li, Y. et al. Comparative proteomic analyses using iTRAQ-labeling provides insights into fiber diversity in sheep and goats. Journal of Proteomics. 172, 82-88 (2017).

61. Zhou, Y. Y. et al. Increased expression of KCTD9, a novel potassium channel related gene, correlates with disease severity in patients with viral hepatitis B. Chinese journal of hepatology. 16, 835 (2008).

62. Chen, T. et al. KCTD9 contributes to liver injury through NK cell activation during hepatitis B virus-induced acute-on-chronic liver failure. Clinical Immunology. 146, 207-216 (2013).

63. Zhang, X. et al. Interference with KCTD9 inhibits NK cell activation and ameliorates fulminant liver failure in mice. BMC Immunology. 19, 20 (2018).

64. Dong, Y. et al. Sequencing and automated whole-genome optical mapping of the genome of a domestic goat (Capra hircus). Nature Biotechnology. 31, 135-141 (2012).

65. Trapnell, C. et al. Differential gene and transcript expression analysis of RNA-seq experiments with TopHat and Cufflinks. Nature Protocols. 7, 562-578 (2012)

66. Pertea, M., Kim, D., Pertea, G. M., Leek, J. T. \& Salzberg, S. L. Transcript-level expression analysis of RNA-seq experiments with HISAT, StringTie and Ballgown. Nature Protocols. 11, 1650-1667 (2016).

67. Zhang, X. O. et al. Diverse alternative back-splicing and alternative splicing landscape of circular RNAs. Genome Res. 26, 1277-1287 (2016).

68. Robinson, M. D., Mccarthy, D. J. \& Smyth, G. K. edgeR: a Bioconductor package for differential expression analysis of digital gene expression data. Bioinformatics. 26, 139-140 (2014).

69. Frazee, A. C. et al. Ballgown bridges the gap between transcriptome assembly and expression analysis. Nature Biotechnology. 33, 243-246 (2015)

70. Xie, C. et al. Kobas 2.0: a web server for annotation and identification of enriched pathways and diseases. Nucleic Acids Research. 39, W316-W322 (2011).

71. Kanehisa, M., Furumichi, M., Tanabe, M., Sato, Y. \& Morishima, K. KEGG: new perspectives on genomes, pathways, diseases and drugs. Nucleic Acids Research. 45, D353-D361 (2017).

72. Agarwal, V., Subtelny, A. O., Thiru, P., Ulitsky, I. \& Bartel, D. P. Predicting microRNA targeting efficacy in Drosophila. Genome Biol. 19, 152 (2018).

73. Saito, R. et al. Atravel guide to Cytoscape plugins. Nat. Methods. 9, 1069-1076 (2012).

74. Li, J., Li, Q., Chen, L., Gao, Y. \& Li, J. Expression profile of circular RNAs in infantile hemangioma detected by RNA-Seq. Medicine. 97, e10882 (2018).

75. Livak, K. J. \& Schmittgen, T. D. Analysis of Relative Gene Expression Data Using Real-Time Quantitative PCR and the $2^{-\Delta \Delta C T}$ Method. Methods. 25, 402-408 (2001). 


\section{Acknowledgements}

The work was supported financially by grants from the National Natural Science Foundation of China (No. 31802038, 31872325, 31672388), Natural Science Foundation of Liaoning Province, China (No. 2015020758), Key Project Foundation of Education Department of Liaoning Province, China (No. LSNZD201606), Breeding project of new Liaoning cashmere goat "meat and meat dual-use" (No. 2017202005), Science and Technology Innovation Talent Support Foundation for Young and Middle-aged People of Shenyang City, China (RC170447).

\section{Author contributions}

Data curation, Yuanyuan Zheng; Formal analysis, Yuanyuan Zheng and Chang Yue; Funding acquisition, Zeying Wang and Wenlin Bai; Methodology, Suping Guo, Zeying Wang and Wenlin Bai; Project administration, Wenlin Bai; Resources, Dan Guo and Suling Guo; Software, Yuanyuan Zheng and Taiyu Hui; Supervision, Zeying Wang; Validation, Yuanyuan Zheng and Jiaming Sun; Writing-original draft, Yuanyuan Zheng; Writing-review \& editing, Yuanyuan Zheng, Bojiang Li, Zeying Wang and Wenlin Bai.

\section{Competing interests}

The authors declare no competing interests.

\section{Additional information}

Supplementary information is available for this paper at https://doi.org/10.1038/s41598-019-57404-9.

Correspondence and requests for materials should be addressed to Z.W. or W.B.

Reprints and permissions information is available at www.nature.com/reprints.

Publisher's note Springer Nature remains neutral with regard to jurisdictional claims in published maps and institutional affiliations.

Open Access This article is licensed under a Creative Commons Attribution 4.0 International License, which permits use, sharing, adaptation, distribution and reproduction in any medium or format, as long as you give appropriate credit to the original author(s) and the source, provide a link to the Creative Commons license, and indicate if changes were made. The images or other third party material in this article are included in the article's Creative Commons license, unless indicated otherwise in a credit line to the material. If material is not included in the article's Creative Commons license and your intended use is not permitted by statutory regulation or exceeds the permitted use, you will need to obtain permission directly from the copyright holder. To view a copy of this license, visit http://creativecommons.org/licenses/by/4.0/.

(C) The Author(s) 2020 